\title{
Identifying and Characterizing Cyclicality and Seasonality in Environmental Data with An Adaptive Filter Implementation of A Phase Locked Loop
}

\author{
J. D. Linton ${ }^{1,2}$ and E. Daw ${ }^{3 *}$ \\ ${ }^{1}$ School of Management, University of Sheffield Sheffield, Sheffield S10 2TN, United Kingdom \\ ${ }^{2}$ Institute for Statistical Studies and Economics of Knowledge, Higher School of Economics, Moscow 101000, Russia \\ ${ }^{3}$ Department of Physics, University of Sheffield, Sheffield S10 2TN, United Kingdom
}

Received 07 April 2020; revised 15 June 2020; accepted 20 June 2020; published online 31 December 2020

\begin{abstract}
Phase Locked Loops are provided as a manner of assessing, monitoring and forecasting with seasonal or cyclical data. Phase Locked Loops using adaptive filtering were initially utilized for gravitational wave data analysis. In this paper we examine this implementation as an alternative approach for identifying the presence of one or more cycles in environmental data. Unlike most techniques for assessing seasonality phase lock loops can identify the presence of cycles without prior suspicion of their nature and existence. This is important for identifying either the initiation of new cycles or changes in existing cycles. As environmental data can be impacted by the presence of numerous cyclical phenomena and these cycles can change over time, this is a suitable and valuable technique. The technique can be used on existing data sets or assessing data streams. Phase Locked Loops are advantageous over many currently as the presence of multiple different cycles can be identified without prior suspicion and specification of the nature of the cyclicality of the data. The speed and parsimonious nature of the technique makes Phase Lock Loops ideal for Big Data and data flows that are so large that they must be assessed on a real time basis. The technique can manage and advise of changes in frequency, amplitude and phase shifts. The technique is illustrated utilizing weather data.
\end{abstract}

Keywords: phase lock loops, forecasting, seasonality, cycles

\section{Introduction}

This paper addresses the need for techniques that are more adept at identifying the presence of cycles in existing environmental data bases and monitoring cycles in real time data feeds. In addition to considering the dimensions that are important to selection of the appropriate technique for cyclicality detection, monitoring and forecasting for a given application; the Phase Locked Loop is introduced and demonstrated as a tool for environmental informatics. The Phase Locked Loop is valuable for applications that benefit from parsimony, responsiveness and ease of use. The application of the Phase Locked Loop as a technique for assessing cyclical patterns in data is illustrated through the consideration of weather data. As the technique can identify change in frequency and amplitude, phase transitions and the emergence/disappearance of new cycles, the technique has tremendous flexibility that is not captured by the example.

Determining presence of cycles is not only important for the fundamental understanding of systems, but also for prescriptive reasons such as more accurate forecasting. The existence and investigation of the presence of cyclical behavior is common in many fields primarily due to weather related ef-

* Corresponding author. Tel.: +44 1142224353.

E-mail address: e.daw@sheffield.ac.uk (E. Daw).

ISSN: 2663-6859 print/2663-6867 online

(C) 2020 ISEIS All rights reserved. doi:10.3808/jeil.202000042. fects, such as: flooding (Atiya et al., 1999; Marchi et al., 2010), climate change (Keating et al., 2003; Battisti and Naylor, 2009), and health related issues (D'Amato et al., 1998; Thomson et al., 2006). Often seasonality is used as an alternate nomenclature to cyclicality as a cycle is often defined by events natural and/or social - that occur in a single calendar year. A variety of criteria are used for determining which technique is most suitable for measuring cyclicality, based on the ability to best satisfy the application under consideration. For measuring cycles, performance criteria include: (1) parsimony (how many cycles are required to identify a pattern), (2) responsiveness (the delay before a change in the cycle be identified), (3) resources (how much computing power is required for determination, monitoring, and/or prediction), (4) ease of use (what level of skill is required by user). These four criteria are best considered on a case-by-case basis to offer guidance on which technique is best used for a given application.

Cyclicality is commonplace in environmental analytics examples include air quality (Zhu et al., 2017), algal blooms (Lui et al., 2007), dissolved oxygen (Díaz and Rosenberg, 2011; Lee et al., 2013), disease outbreaks (Wang and Deng, 2012), hydrology (Banerjee et al., 2009; Svensson et al., 2015), ozone levels (Randriambelo et al., 2000), salinity (Hackert, et al., 2011), solar power (Lin and Pai, 2016), species cyclicality (Lloret et al., 2000; Jaksic et al., 2003), water temperature (Neetu et al., 2012), water quality (DiDonato et al., 2009), wave power (Ayat, 
2013; Rusu and Onea, 2013), weather (De Felice et al., 2015; Lusis, Khalilpour et al., 2017), and wind (Marvuglia and Messineo, 2012). For these applications a variety of techniques can be utilized, including: seasonal decomposition, exponential smoothing methods, autoregressive integrative moving average models, transforms, grey forecasting, machine learning, kernel density and integrated techniques. The simplest case is that seasonality is suspected and the length of the cycle is known usually daily, weekly or annual fluctuations - a Seasonal Moving Average (SMA) can be calculated. The SMA is removed through the process of seasonal decomposition (de Livera et al., 2011) to examine the behavior of the data once the seasonal effect has been removed. This technique requires a recognition, or suspicion, of the presence of periodicity in the data under consideration. There are other techniques that assist in identifying suspected seasonal variation.

Holt Winters (triple exponential smoothing) allows for the detection and forecasting of both chronological and seasonal patterns (Taylor, 2008; Ilbeigi et al., 2017). Furthermore, AutoRegressive Integrative Moving Average (ARIMA) models can be applied to detect seasonality of a known period - the Seasonal AutoRegressive Integrative Moving Average (Ediger and Akar, 2007; Schulzea and Prinzb, 2009). In fact, Box Jenkins (Box et al., 2013) can also be used for this purpose. Once again the length of the period must be defined.

However, there are transforms that can detect the presence and periodicity of waves without prior knowledge of their existence. While these approaches are helpful as they require no prior knowledge, more time, expertise and computational power is required. While the Fourier and fast Fourier (Martens et al., 2002; de Livera et al., 2011) transforms are most frequently mentioned, other transforms such as wavelets (Choi et al., 2011; Bessec and Fouquau, 2018) and the Box-Cox transform (de Livera et al., 2011; Petropoulos et al., 2018) have also been utilized for wave characterization and forecasting.

In addition, there are lesser used techniques such as Grey forecasting (Tseng et al., 2001; Xia and Wong, 2014) which is useful for dealing with seasonality when analysis is limited by very small data sets. Neural nets, machine learning and artificial intelligence (Chu and Zhang, 2003; Venkatesh et al., 2014; Erişen et al., 2017) have also been utilized for wave characterization and forecasting. Kernel density (Arora and Taylor, 2016) has also been used for detecting and modelling seasonality in data streams. Finally, some researchers have proposed the integration of two different techniques to model seasonality. These combinations include: applying statistical and machine learning (Tong and Liang, 2005) and evolutionary seasonal decomposition using a least-square support vector regression (ESDLS-SVR) (Lin and Pai, 2016).

Applications involving the detection, measurement, monitoring and forecasting of cyclicality can benefit from the performance characteristics of Phase Locked Loops. Phase Locked Loops are ubiquitous workhorses in engineering and measurement with applications as diverse as automatic control (Hsieh and Hung, 1996), communications (Kazovsky et al., 2006), gravitational wave detection (Aasi et al., 2015), and ato- mic force microscopy (Ruppert et al., 2016).

The Phase Lock Loop is demonstrated as a technique for identifying and assessing cyclicality on temperature data for the Chicago O'Hare Airport. Temperature is an example of an environmental phenomena that involves multiple cycles occurring simultaneously - a daily and annual cycle. For many airports temperature readings that are both accurate and frequent are available for an extended period of time. Consequently, the data set is appropriate for demonstrating and testing a technique that sets out to identify, characterize and track cycles.

Phase Locked Loops have been selected for applications, due to a strong fit between the performance characteristics and the needs of the application. In all cases, applications have benefited from some combination of the Phase Locked Loops performance characteristics. (1) Parsimony - Phase Locked Loops can quickly identify the underlying cycle(s) pattern based on either user specification or an automated search routine. (2) Responsiveness - the Phase Locked Loop can quickly identify: changes in frequency, phase-shift of the cycle, or the appearance/disappearance of a cyclical pattern (3) Resources - the Phase Locked Loop requires much fewer calculations compared to many other techniques - such as fast Fourier transforms. When using a Fourier Transform to track a wave having a relatively stationary frequency, the computation time associated with an Fast Fourier Transform of $\mathrm{N}$ points of data is order N*log_2(N), where log_2 is the base-2 log. The Phase Lock Loop utilizes $\mathrm{N}$ operations to analyse $\mathrm{N}$ data points. In other words, it is a factor of $\log \_2(\mathrm{~N})$ faster than the Fourier Transform. In the case of 256 data points, the Phase Lock Loop is about 8 times faster and for 65,536 data points the Phase Lock Loop is about 16 times faster. Consequently, the initial processing and continued monitoring time is low. This is important if reduced computing resources or energy consumption is considered important. (4) Ease of use - is provided as it is dependent on an already existing algorithm (Daw and Edo, 2016). The search for and monitoring of multiple cycles simultaneously can be automated. However, the option of the analyst using a Phase Locked Loop to interrogate data regarding the existence of specific cycles is also possible. The former approach requires more initial set-up of the algorithm, but low skill requirements for continued use, due to automation. While the latter approach requires, a more sophisticated user.

In the case of environmental informatics, the Phase Lock Loop is notable in that: (1) Easy to use - there is no need for a sophisticated knowledge of either mathematics or statistics to interpret the output. (2) Directed (theoretical) or nondirected (data-driven) use - can either verify the existence of a suspected cycle(s) or scan for the presence of unexpected cycles. For example, second-order effects. (3) Flexible - changes in amplitude, frequency or phase shifts are quickly identified and the Phase Lock Loop adjusts to continue tracking. While not valuable for the weather example given in this paper, in the case of Levi flights or the impact of an unusual events an understanding of the presence and nature of change is valuable. (4) $R e$ source efficient - as low computational power is required for real time data analysis the Phase Lock Loop is useful for infield analysis where energy and computational power is limited. 
The technique is also appropriate for assessment of very rapid data streams. Having considered the techniques typically used to assess cyclicality in business/management applications and summarized the performance characteristics of Phase Locked Loops, the Phase Locked Loop technique is considered.

\section{Method}

For detection and tracking of oscillations related to seasons and cyclicality, a variant of the Phase Locked Loop technique (Gardner, 2005) is used. A brief description of the method is presented before consideration of application to real world problems. Consider input data consisting of a stream $x_{n}$ of real numbers, where $n$ is an integer that increases with time. The data consists of samples from an oscillation, $X_{n}=A_{n} \cos$ $\left(2 \pi f_{n} \tau_{n}+\phi\right)+S_{n}$. Here, $A_{n}$ and $f_{n}$ are the amplitude and frequency of the wave, $\phi$ is a phase offset at time zero, $\tau_{n}$ is the time when the $n^{\text {th }}$ sample is acquired, and $S_{n}$ is the background (Proakis and Manolakis, 1996), a combination of any remaining oscillations plus further broadband contributions such as noise. All parameters with an $n$ subscript may exhibit sample-to-sample variations, and it is the task at hand to deduce best estimates for these quantities. It is sometimes true that the data is regularly sampled, in which case we may write $\tau_{n}=n \tau_{s}$, where $\tau_{s}$ is a constant sampling period, the reciprocal of the sampling rate $f_{s}$.

The input data stream is applied to the following iteration algorithm:

$$
\left(\begin{array}{c}
y_{n}^{R} \\
y_{n}^{I}
\end{array}\right)=(1-w)\left(\begin{array}{ll}
\cos \Delta_{n} & -\sin \Delta_{n} \\
\sin \Delta_{n} & \cos \Delta_{n}
\end{array}\right)\left(\begin{array}{l}
y_{n-1}^{R} \\
y_{n-1}^{I}
\end{array}\right)+w\left(\begin{array}{l}
x_{n} \\
0
\end{array}\right)
$$

The parameter $w$ is a number in the range $(0,1)$ that determines a response time $\tau$ for the iteration algorithm to changes in the amplitude of the wave. The parameter $\Delta_{n}$ is the phase shift between the current and previous samples, so that $\Delta_{n}=2$ $\pi f_{n}\left(\tau_{n}-\tau_{n-1}\right)$. In the case of regularly sampled data, $w$ is given by $w=\tau_{s} / \tau$ and $\Delta_{n}=2 \pi f_{n} \tau_{s}$. Output from this iteration algorithm is then further processed through matrix multiplication, resulting in two streams of processed data, referred to here as $D_{n}$ and $Q_{n}$, as follows:

$$
\left(\begin{array}{l}
D_{n} \\
Q_{n}
\end{array}\right)=\left(\begin{array}{cc}
T_{11} & T_{12} \\
T_{21} & T_{22}
\end{array}\right)\left(\begin{array}{c}
y_{n}^{R} \\
y_{n}^{I}
\end{array}\right)
$$

where the elements of the matrix, $T_{a b}$ are functions of the parameters $w$ and $\Delta_{n}$. When the tracking algorithm is 'locked' onto the oscillation, $f_{n}$ is an estimate of the frequency of the oscillation, and the streams $D_{n}$ and $Q_{n}$ form oscillations at the same frequency as the input oscillation being tracked. The contribution of the support $S_{n}$, is suppressed by a factor dependent on $w$. The 'direct' component $D_{n}$ is in phase with the oscillation being tracked, and the 'quadrature' component $Q_{n}$ lags the input oscillation in phase by $90^{\circ}$. The algorithm therefore forms an orthogonal state generator (Daw and Edo, 2016) for the input oscillation. Orthogonal State Generators (OSGs) are important since it is the orthogonal state (a wave at the same frequency as the cycle in the data but out-of-phase with it), that is used to detect changes in frequency and phase. Other researchers have also developed OSGs using different techniques; see for example (Ciobotaru and Blaabjerg, 2006).

The quantity $E_{n}=\left(x_{n}-D_{n}\right) Q_{n} / A_{n}$ is employed as an error signal (Franklin et al., 1994) to sense departures of the frequency of the oscillation from the previously estimated value $f_{n}$ The updated value of the frequency for the next iteration of the algorithm is given by:

$f_{n+1}=G E_{n}+f_{n-1}$

where $G$ is a constant coefficient chosen so that the feed-back circuit adjusting the frequency is a second order loop critically damped with respect to step changes in cyclicity (Gardner, 2005). The amplitude of the wave is given by $A_{n}=\sqrt{D_{n}^{2}+D_{n}^{2}}$. With the frequency tracking loop locked, the best estimate of the support, or the data with the tracked oscillation removed, is $S_{n}=x_{n}-D_{n}$. Further oscillations forming part of the support can be tracked by using more than one loop applied to the data either in parallel or in series. This procedure can be applied to identify and simultaneously track numerous oscillations in the data, as has been in the case in the initial application the detection of gravitational waves (Abbott et al., 2016).

The algorithm utilizes an adaptive resonant filter to detect and track wave components of arbitrary data. A single free parameter controls the time the filter takes to locate and lock on to a wave, the response time of the filter to changes in amplitude or frequency, and the bandwidth of the algorithm for including spectral features at neighboring frequencies. Multiple instances of the filter can run in parallel. The filter output can include a de-noised facsimile of the sine wave which can be subtracted from the data input to other instances of the filter. This cross-subtraction technique is used to break degeneracies between instances following sine waves whose frequencies are close together.

The phase-lock loop is initialized with an initial guess frequency. In the absence of intervening spectral frequencies, the approach of the frequency to the actual line frequency is exponential, with a time constant equal to twice the input timescale parameter, which is referred to as $\tau$. If the sine wave subsequently undergoes a step change in amplitude, the response to the change in amplitude is also exponential, and the time constant for the exponential approach is also $\tau$. A longer $\tau$ is associated with a narrower bandwidth $B$, related to $\tau$ by $B=1$ / $(\pi \tau)$.

Higher bandwidth means that the frequency and amplitude estimate on the line from the algorithm will be noisier. There is therefore a trade-off between responsiveness and accuracy. A larger $\tau$ means it will take longer to acquire the correct sine wave parameters, but once acquired the parameters reconstructed will be more accurate, as long as the sine wave is sufficiently stable. A shorter $\tau$ will result in a more reactive tracking algorithm, but the reconstructed parameters will be less ac- 
curate. This is a challenge for data in management and business fields as it tends to be noisy and have a small number of cycles.

\section{Operationalizing Phase Locked Loops}

It is worth summarizing key characteristics that separate this technique from many of the other approaches to modelling, monitoring and forecasting cyclical data:

(1) Ability to identify and track multiple waves simultaneously - due to the high speed and low computing power requirements it is possible to search for and/or track many waves at the same time. The technique has successfully identified and tracked 15 separate waves simultaneously.

(2) Ability to identify and track suspected waves - a specific period can be identified. Once lock on occurs, the Phase Locked Loop will adapt to phase shifts and changes in period. The system is able to report changes in behavior and identify instantaneous and ongoing deviations from expected behavior.

(3) Ability to search for waves when the period is unknown. This includes the ability to search for and detect the onset of new waves; the original purpose of the implementation of Phase Locked Loop algorithms described in this paper was to characterize and remove instrumental oscillations that were obscuring the signals from collisions of black holes (Abbott et al., 2016) and neutron stars (Abbott et al., 2017) in the Laser Interferometer Gravitational-wave Observatory (LIGO) gravitational wave detectors.

(4) Ability to be used on big data problems involving the assessment of databases with large numbers of variables and observations, due to its high speed and low computing power requirements

(5) Ability to be used on data problems that are so big that data is analyzed and summarized on a real time basis. This eliminates the need for data storage and provides insights onto changes in periodicity and behavior on a real time basis - delay at the millisecond order of magnitude. Important for management of real time data feeds.

Having summarized the noteworthy features of Phase Locked Loops for identifying, monitoring and forecasting periodicity, an assessment of weather data is now offered as an exemplar.

For the purpose of demonstrating and/or testing a technique that assesses, monitors and/or predicts cyclical behavior, the dynamics of temperature change is useful as temperature has both a daily cycle and an annual cycle. Also accurate temperature data is readily available. For the purpose of illustration, weather data for a 10-year period for Chicago O-Hare airport was utilized (NOAA, 2017). The readings occur at least once an hour over non-even intervals (minimum and maximum intervals are 6 and 60 minutes). Consequently, the algorithm cannot place each reading into a separate bin of equal size for the purpose of simplifying calculation. Chicago O'Hare has a continental climate but is influenced by its proximity to a large body of water - Lake Michigan - that can have a warming effect on the cold winds that blow from the north during the winter.
The effect of sunlight on daily temperature gain is affected by the increased presence of overcast skies at times of the year in which the air temperature is cooling at a faster rate than the water temperature (typical in the fall and winter).

The Phase Locked Loop algorithm was set to an initial assumption of two separate cycles with a duration of one day and one year. Results are provided in Figures 1 to 6.

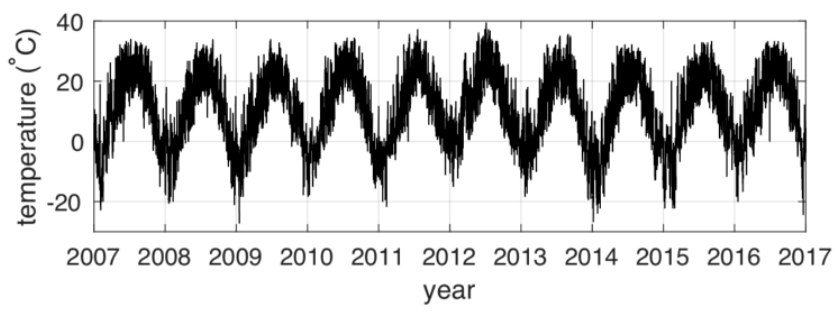

Figure 1. Temperature for Chicago-O'Hare Airport 2007 2017, inclusive (Source (NOAA, 2017)).

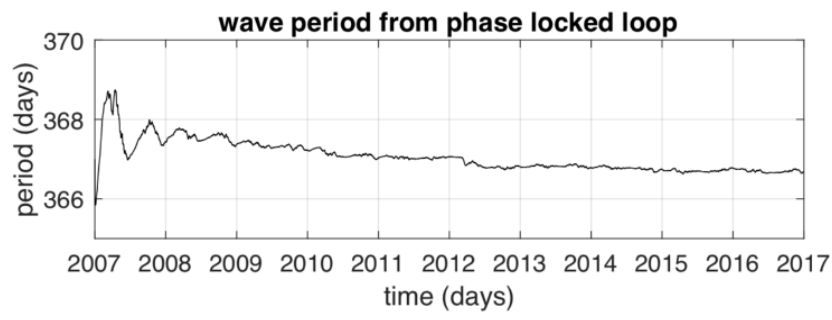

Figure 2. Length of yearly period (cycle 1) estimated dynamically by the Phase Locked Loop algorithm

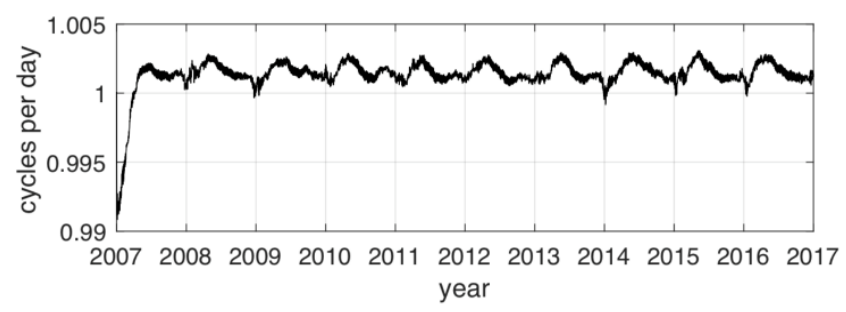

Figure 3. Length of daily period (cycle 2) estimated dynamically by the Phase Locked Loop algorithm.

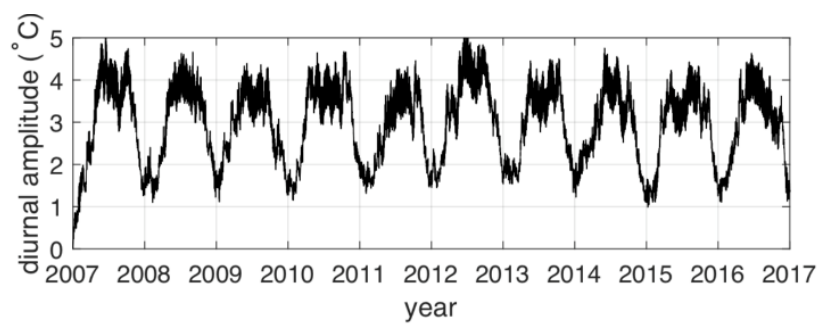

Figure 4. Diurnal amplitude of the cycle estimated dynamically by the Phase Locked Loop algorithm. Initially amplitude is underestimated.

The temperature variation is clearly cyclical with temperature near the end/beginning of the year much cooler - reaching lows of -20 degrees Celsius or less. While summer highs are 
well over 30 degrees Celsius (Figure 1). The duration of the annual cycle quickly increases to over 368 days, but then attenuates at a value under 367 days (Figure 2). Similarly, the duration of the daily cycle quickly increases and slightly overshoots the day duration never going beyond 1.002 days (Figure 3). However, unlike the annual cycle measurement, the daily cycle measurement shows evidence of an annual pattern with slight increases and decreases over time. Figure 4 illustrates the diurnal amplitude of the temperature during the period under consideration. The amplitude drops to close to 1 degree Celsius at the end/start of a year and increases to about 4 degrees Celsius for the summer period. Figures 5 and 6 consider the error for the yearly and daily cycle, respectively. Error is much higher at the start of the training period, reflecting the instability noted on the other graphs. Also worth noting is that the error is much higher when considering the cyclical prediction of daily data, rather than that of annual data. Having provided two illustrations of cyclical data - mortality (a single annual cycle) and temperature (a daily and an annual cycle). The implications that this technique has to theory and practice is now considered.

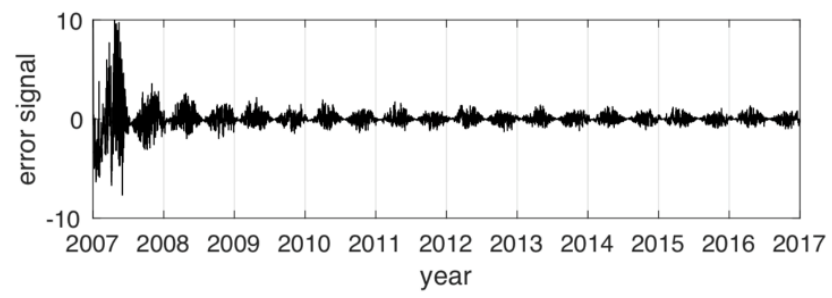

Figure 5. Measure of error for annual cycle estimated dynamically based on Phase Locked Loop estimation of the appropriate value.

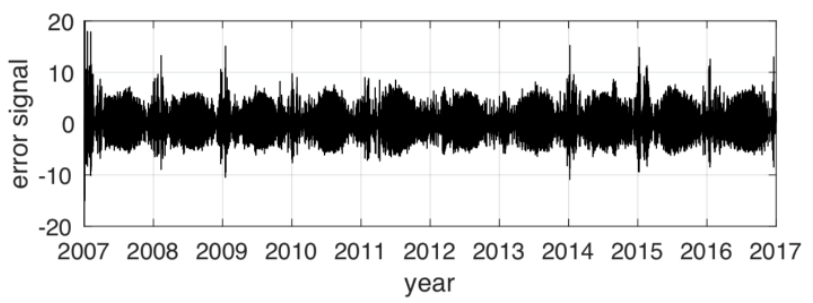

Figure 6. Measure of error for daily cycle estimated dynamically based on Phase Locked Loop estimation of the appropriate value.

\section{Discussion}

\subsection{Implications to Theory}

The use of Phase Locked Loops for assessing the presence of one or more cyclical patterns is an example of where a technique developed for the unique needs of fundamental research potentially provides many applied benefits. While the opportunity to harvest non-scientific value from fundamental research are abundant, they are often overlooked. In this particular case, the unusual fundamental science requirement is the tracking and filtering out a large number of waves from a sensitive detection device so that the wave resulting from the conversion of matter into energy (empirical validation of the relation $E=m c^{2}$ ) as a result of either the collision of black holes or neutron binary stars can be recognized and characterized. While this fundamental contribution is worthwhile on its own as an important scientific discovery including being awarded the 2017 Nobel Prize in Physics, seemingly unusual requirements associated with this project and other fundamental science activities can also provide significant benefits to better characterizing, monitoring, and forecasting environmental data.

As a technique it offers some significant advantages. Like transforms or machine learning, Phase Locked Loops do not require that the analyst has prior knowledge or suspicion of the nature of the cyclicality that may exist in the data. Similarly, the Phase Locked Loop can identify multiple cycles in a single data set. However, this is accomplished with the use of much lower computational power. The result is a fast and parsimonious use of processing power allowing for the exploration of large data sets for relationships that may provide useful insights.

If the analyst has a suspicion of the presence of cyclical behavior, initial estimates can be set to reflect these perceptions resulting in faster convergence. However, the analyst can also set a number of Phase Locked Loops to actually search for the either presence of unanticipated cycles or the initiation and emergence of new cycles. As the Phase Locked Loop locks onto a cycle, all changes in the cycle are reported: amplitude, phase shifts, changes in frequency and cycle termination. In this respect, the Phase Locked Loop is superior to other techniques used to model and forecast cyclical behavior in business, management and related fields. While the Phase Locked Loop is not being proposed as a replacement for the existing techniques used to measure seasonality and cyclicality, it is an important discovery as its parsimonious nature makes it ideal for big data and/or continuous data streams.

\subsection{Implications to Practice}

As the availability of inexpensive sensors and memory increases, the challenge of data overload is increasingly problematic. Consequently, techniques that can be used to assess and find relationships are of increasing importance. Especially important are techniques that require minimal time and/or computing power.

The movement towards the interconnection of equipment and sensors through the Internet of Things is further increasing the real time flow of data. For many applications the detection and monitoring of cycles in situ or remotely can be of assistance.

\section{Conclusion}

Phase Locked Loops offer a parsimonious flexible technique to identify the presence of one or more cycles in a data stream. Through its initial use in identifying gravitational waves resulting from the collision of two black holes, the technique has been found to be able to identify, characterize and track 15 cycles simultaneously. In addition to identifying phase shifts, changes in frequency, changes in amplitude, the Phase Locked 
Loop can identify the emergence of additional cycles in a data stream. The Phase Locked Loop has been demonstrated on weather data illustrating the speed of convergence and the nature of the output. As Phase Locked Loops can respond to changes in the nature of a cycle that occurs in less than a millisecond, the technique can not only be used for the relatively static data sets utilized as examples, but also for dynamic real time data.

Acknowledgements. Support was received from the Higher Education Innovation Fund administered by the University of Sheffield through the grant Understanding the Pathways and Easing the Journey for Externalizing Algorithms. This study was conducted within the framework of the Basic Research Program at the National Research University Higher School of Economics (HSE) and supported within the frame-work of implementation of the HSE 5-100 Program Roadmap.

\section{References}

Aasi, J., Abbott, B.P., Abbott, R., Abbott, T., and Zweizig, S. (2015). Advanced LIGO. Classical and Quantum Gravity, 32(7). https://doi: 10.1088/0264-9381/32/7/074001

Abbott, B.P., Abbott, R., Abbott, T.D., Abernathy, M.R., Acernese, F., Ackley, K., and Zweizig, J. (2016). Observation of gravitational waves from a binary black hole merger. Physical Review Letters, 116(6), 16. https://doi:10.1103/Phys RevLett. 116.061102

Abbott, B.P., Abbott, R., Abbott, T.D., Acernese, F., Ackley, K., Adams, C., and Zweizig, J. (2017). GW170817: Observation of Gravitational Waves from a Binary Neutron Star Inspiral. Physical Review Letters, 119(16), 18. https://doi:10.1103/PhysRevLett.119. 161101

Arora, S. and Taylor, J.W. (2016). Forecasting electricity smart meter data using conditional kernel density estimation. Omega, 59, 47-59. https://doi:10.1016/j.omega.2014.08.008

Atiya, A.F., El-Shoura, S.M., Shaheen, S.I., and El-Sherif, M.S. (1999). A comparison between neural-network forecasting techniques Case study: River flow forecasting. IEEE Transactions on Neural Networks, 10(2), 402-409. https://doi: 10.1109/72.750569

Ayat, B. (2013). Wave power atlas of Eastern Mediterranean and Aegean Seas. Energy, 54, 251-262. https://doi:10.1016/j.energy.2013. 02.060

Banerjee, P., Prasad, R.K., and Singh, V.S. (2009). Forecasting of groundwater level in hard rock region using artificial neural network. Environmental Geology, 58(6), 1239-1246. https://doi:10.1007/ s00254-008-1619-z

Battisti, D.S. and Naylor, R.L. (2009). Historical warnings of future food insecurity with unprecedented seasonal heat. Science, 323 (5911), 240-244. https://doi:10.1126/ science.1164363

Bessec, M. and Fouquau, J. (2018). Short-run electricity load forecasting with combinations of stationary wavelet transforms. European Journal of Operational Research, 264(1), 149-164. https://doi: 10.1016/j.ejor.2017.05.037

Box, G.E.P., Jenkins, G.M., and Reinsel, G.C. (2013). Time series analysis: Forecasting and control: Fourth edition. New York: John Wiley and Sons.

Choi, T.M., Yu, Y., and Au, K.F. (2011). A hybrid SARIMA wavelet transform method for sales forecasting. Decision Support Systems, 51(1), 130-140. https://doi:10.1016/j.dss. 2010.12.002

Chu, C.W. and Zhang, G.P. (2003). A comparative study of linear and nonlinear models for aggregate retail sales forecasting. International Journal of Production Economics, 86(3), 217-231. https:// doi:10.1016/S0925-5273(03)00068-9

Ciobotaru, M., Teodorescu, R., and Blaabjerg, F. (2006). A new single- phase PLL structure based on second order generalized integrator. IEEE Power Electronics Specialists Conference, PESC '06. 37th IEEE, 1-6.

D'Amato, G., Spieksma, F.T.M., Liccardi, G., Jäger, S., Russo, M., Kontou-Fili, K., Nikkels, H., Wüthrich, B., and Bonini, S. (1998). Pollen-related allergy in Europe. Allergy: European Journal of Allergy and Clinical Immunology, 53(6), 567-578. https://doi:10.11 11/j.1398-9995.1998.tb03932.x

Daw, E. and Edo, T. (2016). Parameter Estimation and Control Method and Apparatus. International Patent Organization: Filing Number, WO2016128775.

De Felice, M., Alessandri, A., and Catalano, F. (2015). Seasonal climate forecasts for medium-term electricity demand forecasting. Applied Energy, 137, 435-444. https://doi:10.1016/j.apenergy.2014. 10.030

De Livera, A.M., Hyndman, R.J., and Snyder, R.D. (2011). Forecasting time series with complex seasonal patterns using exponential smoothing. Journal of the American Statistical Association, 106(496), 1513-1527. https://doi:10.1198/jasa.2011.tm09 771

Díaz, R.J. and Rosenberg, R. (2011). Introduction to environmental and economic consequences of hypoxia. International Journal of Water Resources Development, 27(1), 71-82. https://doi:10.1080/ 07900627.2010.531379

DiDonato, G.T., Stewart, J.R., Sanger, D.M., Robinson, B.J., Thompson, B.C., Holland, A.F., and Van Dolah, R.F. (2009). Effects of changing land use on the microbial water quality of tidal creeks. Marine Pollution Bulletin, 58(1), 97-106. https://doi:10.1016/j.mar polbul.2008.08.019

Ediger, V.S. and Akar, S. (2007). ARIMA forecasting of primary energy demand by fuel in Turkey. Energy Policy, 35(3), 1701-1708. https://doi:10.1016/j.enpol.2006.05.009

Erişen, E., Iyigun, C., and Tanrısever, F. (2017). Short-term electricity load forecasting with special days: an analysis on parametric and non-parametric methods. Annals of Operations Research, 1-34. https://doi:10.1007/s10479-017-2726-6

Franklin, G.F., Powell, J.D., and Emami-Naeini, A. (1994). Feedback Control of Dynamic Systems. New York: Addison Wesley.

Gardner, F.M. (2005). Phaselock Techniques (3rd Ed.). New York: Wiley Interscience.

Hackert, E., Ballabrera-Poy, J., Busalacchi, A.J., Zhang, R.H., and Murtugudde, R. (2011). Impact of sea surface salinity assimilation on coupled forecasts in the tropical Pacific. Journal of Geophysical Research: Oceans, 116(C5). https://doi:10.1029/2010JC006708

Hsieh, G.C. and Hung, J.C. (1996). Phase-locked loop techniques - A survey. IEEE Transactions on Industrial Electronics, 43(6), 609615. https://doi:10.1109/41.544547

Ilbeigi, M., Ashuri, B., and Joukar, A. (2017). Time-Series Analysis for Forecasting Asphalt-Cement Price. Journal of Management in Engineering, 33(1). https://doi:10.1061/(ASCE)ME.1943-5479.000 0477

Jaksic, F.M. and Lima, M. (2010). Myths and facts on ratadas: Bamboo blooms, rainfall peaks and rodent outbreaks in South America. Austral Ecology, 28(3), 237-251. https://doi:10. 1046/j.1442-9993. 2003.01271.x

Kazovsky, L.G., Kalogerakis, G., and Shaw, W.T. (2006). Homodyne phase-shift-keying systems: Past challenges and future opportunities. Journal of Lightwave Technology, 24(12), 4876-4884. https:// doi:10.1109/jlt.2006.883692

Keating, B.A., Carberry, P.S., Hammer, G.L., Probert, M.E., Robertson, M.J., Holzworth, D., Huth, N.I., Hargreaves, J.N.G., Meinke, H., Hochman, Z. (2003). An overview of APSIM, a model designed for farming systems simulation. European Journal of Agronomy, 18(34), 267-288. https://doi:10.1016/ S1161-0301(02)00108-9

Lee, Y.J., Boynton, W.R., Li, M., and Li, Y. (2013). Role of Late Winter-Spring Wind Influencing Summer Hypoxia in Chesapeake Bay. Estuaries and Coasts, 36(4), 683-696. https://doi:10.1007/ 
s12237-013-9592-5

Lin, K.P. and Pai, P.F. (2016). Solar power output forecasting using evolutionary seasonal decomposition least-square support vector regression. Journal of Cleaner Production, 134(Part B), 456-462. https://doi:10.1016/j.jclepro.2015.08. 099

Lloret, J., Lleonart, J., and Solé, I. (2000). Time series modelling of landings in Northwest Mediterranean Sea. ICES Journal of Marine Science, 57(1), 171-184. https://doi:10.1006/jmsc. 2000.0570

Lui, G.C.S., Li, W.K., Leung, K.M.Y., Lee, J.H.W., and Jayawardena, A.W. (2007). Modelling algal blooms using vector autoregressive model with exogenous variables and long memory filter. Ecological Modelling, 200(1-2), 130-138. https://doi:10.1016/j.ecolmodel.20 06.06 .017

Lusis, P., Khalilpour, K.R., Andrew, L., and Liebman, A. (2017). Short-term residential load forecasting: Impact of calendar effects and forecast granularity. Applied Energy, 205, 654-669. https:// doi:10.1016/j.apenergy.2017.07.114

Marchi, L., Borga, M., Preciso, E., and Gaume, E. (2010). Characterisation of selected extreme flash floods in Europe and implications for flood risk management. Journal of Hydrology, 394(1-2), 118-133. https://doi:10.1016/j.jhydrol.2010.07.017

Martens, M., Chang, Y.C., and Taylor, S.J. (2002). A comparison of seasonal adjustment methods when forecasting intraday volatility. Journal of Financial Research, 25(2), 283-299. https://doi:10.1111/ 1475-6803.t01-1-00009

Marvuglia, A. and Messineo, A. (2012). Monitoring of wind farms power curves using machine learning techniques. Applied Energy, 98, 574-583. https://doi:10.1016/j.apenergy. 2012.04.037

Neetu, S., Lengaigne, M., Vincent, E.M., Vialard, J., Madec, G., Samson, G., Kumar, M.R.R., and Durand, F. (2012). Influence of upper-ocean stratification on tropical cyclone-induced surface cooling in the Bay of Bengal. Journal of Geophysical Research Oceans, 117(12). https://doi:10.1029/2012JC008433

National Oceanic and Atmospheric Administration (NOAA). (2017). Hourly Output, WBAN: 94846, 2010-2017 inclusive. https://www. ncdc.noaa.gov/

Petropoulos, F., Hyndman, R.J., and Bergmeir, C. (2018). Exploring the sources of uncertainty: Why does bagging for time series forecasting work? European Journal of Operational Research, 268(2), 545. https://doi:10.1016/j.ejor.2018.01.045

Proakis, G. and Manolakis, D. G. (1996). Digital Signal Processing. New York: Prentice Hall

Randriambelo, T., Baray, J.L., and Baldy, S. (2000). Effect of biomass burning, convective venting, and transport on tropospheric ozone over the Indian Ocean: Reunion Island field observations. Journal of Geophysical Research Atmospheres, 105(D9), 11813-11832.
https://doi:10.1029/1999JD901097

Ruppert, M.G., Harcombe, D.M., and Moheimani, S.O.R. (2016). State estimation for high-speed multifrequency atomic force microscopy. American Control Conference, IEEE, 6.

Rusu, E. and Onea, F. (2013). Evaluation of the wind and wave energy along the Caspian Sea. Energy, 50(1), 1-14. https://doi:10.1016/j. energy.2012.11.044

Schulzea, P.M. and Prinzb, A. (2009). Forecasting container transshipment in Germany. Applied Economics, 41(22), 2809-2815. https:// doi:10.1080/00036840802260932

Svensson, C., Brookshaw, A., Scaife, A.A., Bell, V.A., Mackay, J.D., Jackson, C.R., Hannaford, J., Davies, H.N., Arribas, A., and Stanley, S. (2015). Long-range forecasts of UK winter hydrology. Environmental Research Letters, 10(6). https://doi:10.1088/1748-9326/10/ $6 / 064006$

Taylor, J. W. (2008). A comparison of univariate time series methods for forecasting intraday arrivals at a call center. Informs, 54(2), 253265. https://doi:10.1287/mnsc. 1070.0786

Thomson, M.C., Doblas-Reyes, F.J., Mason, S.J., Hagedorn, R., Connor, S.J., Phindela, T., Morse, A.P., and Palmer, T.N. (2006). Malaria early warnings based on seasonal climate forecasts from multimodel ensembles. Nature, 439(7076), 576-579. https://doi:10.1038/ nature 04503

Tong, L.I. and Liang, Y.H. (2005). Forecasting field failure data for repairable systems using neural networks and SARIMA model. International Journal of Quality and Reliability Management, 22(4), 410-420. https://doi:10.1108/02656710 510591237

Tseng, F.M., Yu, H.C., and Tzeng, G.H. (2001). Applied hybrid grey model to forecast seasonal time series. Technological Forecasting and Social Change, 67(2-3), 291-302. https://doi:10.1016/S00401625(99)00098-0

Venkatesh, K., Ravi, V., Prinzie, A., and Van Den Poel, D. (2014). Cash demand forecasting in ATMs by clustering and neural networks. European Journal of Operational Research, 232(2), 383-392. https: //doi:10.1016/j.ejor.2013.07.027

Wang, J. and Deng, Z. (2012). Detection and forecasting of oyster norovirus outbreaks: Recent advances and future perspectives. Marine Environmental Research, 80, 62-69. https://doi:10.1016/j.marenv res.2012.06.011

Xia, M. and Wong, W.K. (2014). A seasonal discrete grey forecasting model for fashion retailing. Knowledge-Based Systems, 57, 119-126. https://doi:10.1016/j.knosys.2013.12.0 14

Zhu, S., Lian, X., Liu, H., Hu, J., Wang, Y., and Che, J. (2017). Daily air quality index forecasting with hybrid models: A case in China. Environmental Pollution, 231, 1232-1244. https://doi:10.1016/j.env pol.2017.08.069 